4 (1) Institute of Solar-Terrestrial Physics SB RAS, 664033, Irkutsk, p/o box 291,126a during solar radio bursts

Yu. V. Yasyukevich ${ }^{1}$, A.S. Yasyukevich ${ }^{1 *}$, E.I. Astafyeva ${ }^{2}$

\title{
How modernized and strengthened GPS signals enhance the system performance
}

5 Lermontov str., Russia

6 (2) Université de Paris, Institut de Physique du Globe de Paris (IPGP), 75013, 35-39 Rue

7 Hélène Brion, Paris, France, e-mail: astafyeva@ipgp.fr

8 Corresponding author: Anna S. Yasyukevich (annpol@iszf.irk.ru)

\section{Introduction}


Global Navigation Satellite Systems (GNSS) have been widely implemented in different fields of industry and transportation. Nowadays, there are several GNSS in operation, such as the American GPS, Russian GLONASS, European Galileo, and the Chinese BeiDou. Therefore, requirements for the accuracy and reliability of GNSS have continuously grown. The performance quality of these systems largely depends on the propagation medium, i.e., on the ionosphere. Irregularities of the ionospheric plasma density may significantly affect radiowave propagation, causing rapid phase and amplitude fluctuations (radio scintillations), or even cause loss of lock (LoL) of satellite signals. These may lead to a significant degradation in system performance, accuracy, and reliability.

Geomagnetic storms are one of the causes of the occurrence of the ionospheric irregularities that lead to the worsening of GNSS performance quality. Afraimovich et al. (2003) showed that the standard deviation in the position determination increased for all types of GPS receivers during magnetic storms. Astafyeva et al. (2014) demonstrated that the number of GPS Losses-of-Lock increases drastically during storms and especially during super-storms. Thus, we are in obvious need of the development of specific space-weather monitoring services that could lead to improving current positioning applications based on GNSS (Jakowski et al. 2005).

A more detailed overview of space weather effects on GNSS performance and ionospheric total electron content (TEC) can be found in Coster and Komjathy (2008) and Afraimovich et al. (2013). A brief review of solar flare effects on the ionosphere is provided in Tsurutani et al. (2009).

Solar radio bursts (SRB) that occasionally accompany solar flares are among the most severe disturbing natural factors for satellite radio technical systems. Klobuchar et al. (1999) predicted that SRBs could affect the GPS performance if the solar flux in the L band frequency range exceeded a threat threshold of about 40,000 solar flux units (sfu). Further studies by Chen et al. (2005) and Demyanov et al. (2013) proved that even weaker solar radio bursts are capable of causing GNSS malfunction. For instance, Chen et al. (2005) showed that almost no GPS L2 signals were tracked around the subsolar area during the October 28, $2003 \mathrm{SRB}$, although the burst flux density at $1415 \mathrm{MHz}$ was only 4,000-12,000 sfu, which is far below the "threat" threshold previously suggested by Klobuchar et al. (1999).

Cerruti et al. (2006) investigated the carrier-to-noise ratio during the September 2005 SRB. They showed that the maximum degradation at GPS L1 reached about $3.0 \mathrm{~dB}$, while at the semi codeless L2 signal degradation of $10.0 \mathrm{~dB}$ was observed. 

sfu solar radio emission the signal-to-noise ratio (SNR) can decrease by 10-30 dB at the navigation receiver antenna. Consequently, the signal tracking losses were detected for a large part of navigation satellites at the earth sunlit part for a rather long time (up to 1 hour). Carrano et al. (2007, 2009) showed that SNR reduction experienced by a GPS receiver depends on the solar incidence angle due to the antenna gain anisotropy.

Muhammad et al. (2015) showed that during the September 24, 2011 extreme SRB event the GPS carrier-to-noise density ratio (C/N0) reduced at most by $13 \mathrm{~dB}$ at L1 and 24 $\mathrm{dB}$ at L2. The $\mathrm{C} / \mathrm{N} 0$ degradation induced the loss of lock at GPS L1 and L2 frequencies and significant code-tracking errors. For the same 2011 SRB event, Sreeja et al. (2013) showed the maximum carrier-to-noise density ratio reductions of $11.0,22.0$, and $10.0 \mathrm{~dB}-\mathrm{Hz}$ for the GPS L1C/A, L2P, and L2C signals, respectively. Degradation in the positioning accuracy in the PPP regime during SRB flares was revealed in Sreeja et al. (2014) and Yasyukevich et al. (2018a).

Sato et al. (2019) pointed out that the degradation of GNSS performance during a flare is frequency dependent. For the September 6, 2017 SRB, the authors recorded the impact of the burst at L2 and L5 frequencies, but not at L1 frequency. The maximum signalto-noise ratio reduction of $-10 \mathrm{~dB}$ was observed while the solar radio flux was pulsating around 2,000 sfu. level. An analysis of the flare impact on the ionosphere and GNSS precise positioning (PPP) and navigation support services has been performed by Berdermann et al. (2018). They revealed that not only the GNSS PPP service was affected during the flare, but also the EGNOS system.

A sudden increase in the total electron content (SITEC) caused by intense EUV flux during solar flares is another potential factor worsening the GNSS in terms of positioning accuracy (Matsoukas et al. 1972). SITEC may dramatically affect the positioning precision by GNSS methods, as was shown in Zhang and Xiao (2005) and Wan et al. (2005). Sudden rapid growth in TEC (and even more importantly, - its second phase derivative) could also potentially result in losses-of-lock. However, only very intensive EUV events, such as the October 28, 2003 flare, when the SITEC exceeded 3 TECU/min (Zhang and Xiao, 2005), can induce a TEC increase high enough to cause LoLs. The September 6, 2017 flare only caused a TEC increase of $\sim 1.5 \mathrm{TECU} / \mathrm{min}$ in subsolar sector (Yasyukevich et al., 2018). The flare induces ionospheric density enhancement depending on solar zenith angle dependent: larger enhancement (and larger SITEC) corresponds to smaller solar zenith angle (Zhang et al. 2019). Previous works also reported that SITEC played a secondary role in losses of signal 
lock (Chen et al., 2005). Rodríguez-Bilbao et al. (2015) concluded that the SITEC was a

100

101

102

103

104

105

106

107

108

109

110

111

112

113

114

115

116

117

118

119

120

122

123 primary source for large errors observed in PPP during the October 28, 2003 flare, while the cycle slips or complete signal loss had been attributed to a SRB that took place on the same day. Sato et al. (2019) and Berdermann et al. (2018) also concluded that the positioning error referred rather to the solar extreme ultraviolet than to solar radio interferences. The authors also noted that a combination of EUV and SRB impact on GNSS signals is possible.

The power of the radio signal emitted by satellites is one of the decisive factors that impact the quality of the estimation of radio navigation parameters. As shown repeatedly (Zakharov et al. 2016; Jin and Oksavik 2018), navigation signal cycle slips occur mainly at the L2 GPS frequency due to two factors: lower transmitter power at L2 and unknown code structure of GPS P(Y) code. To improve this issue, GNSS developers currently upgrade the systems to amend the accuracy and noise-proof features of the systems by improving the ephemeris time support, by increasing the number of navigation signals available to the civilian consumer, and by improving the structure of these signals. Thus, in the GPS system, the satellites have been one-by-one replaced with new-generation modernized GPS satellites (GPS IIR-M, GPS IIF, GPS III) that possess improved characteristics in terms of noise immunity. Another factor in improving system stability in receiver hardware advancements. Fortes et al. (2015) suggested the state-of-the-art acquisition and tracking algorithms that decreases losses of L2 phase lock.

This work analyzes how the change in the emitted GPS satellite signal power affects the system performance stability during solar flares accompanied by intense SRB. In addition, we analyze how new civilian codes can increase the stability of GPS operation during SRB.

\section{GPS signals and their modernization}

Currently, GPS satellites can emit 5 civilian ranging codes: C/A at L1, L2C (M and L codes) at L2, as well as I5 and Q5 codes at L5. The most modern receivers can also semi-codelessly track protected P(Y) signals at L1 and L2. Z-tracking (Ashjaee and Lorenz 1992), which is usually assumed to be the best tracking procedure for the $\mathrm{P}(\mathrm{Y})$ signal. The z-tracking results in equal (or almost equal) signal strength at L1 and L2. However, there is a $13 \mathrm{~dB}$ decrease in signal-to-noise under semi-codeless z-tracking compared to code tracking (HofmannWellenhof et al. 2008). 
According to the GPS interface control document (IS-GPS-200J, 2018), the power of

132

133

134

135

136

137

138

139

140

141

142

143 the GPS signal emitted by the modernized satellite blocks, starting with the IIR-M, was increased to respond to the requirements for the minimum received signal power at a groundbased receiver. Table 1 lists the minimum power values received at ground-based receiver for various types of GPS satellites. One can see that starting with the IIR-M block, the minimum signal power guaranteed by the system at the L2-frequency increased by 3-5 $\mathrm{dBW}$. In addition, for GPS satellites equipped with IIR-M and IIF blocks, there is a possibility to redistribute the transmitting power between the signals.

Table 1 Minimum signal power received at a ground-based receiver (20.46 MHz receiver frequency band). The information is taken from Table 3-Va in IS-GPS-200J, (2018)

\begin{tabular}{|l|c|c|c|}
\hline \multirow{2}{*}{ GPS Block } & \multirow{2}{*}{ Channel } & \multicolumn{2}{|c|}{ Signal } \\
\cline { 2 - 4 } & & P(Y) & C/A or L2C \\
\hline \multirow{2}{*}{ IIA/IIR } & L1 & $-161.5 \mathrm{dBW}$ & $-158.5 \mathrm{dBW}$ \\
\cline { 2 - 4 } & L2 & $-164.5 \mathrm{dBW}$ & - \\
\hline \multirow{2}{*}{ IIR-M/IIF } & L1 & $-161.5 \mathrm{dBW}$ & $-158.5 \mathrm{dBW}$ \\
\cline { 2 - 4 } & L2 & $-161.5 \mathrm{dBW}$ & $-160.0 \mathrm{dBW}$ \\
\hline \multirow{2}{*}{ III } & L1 & $-161.5 \mathrm{dBW}$ & $-158.5 \mathrm{dBW}$ \\
\cline { 2 - 4 } & L2 & $-161.5 \mathrm{dBW}$ & $-158.5 \mathrm{dBW}$ \\
\hline
\end{tabular}

In the current research, we study S1C, S2W, S2X and S5X observables, while the main attention is devoted to the S1C and S2W because P1C and P2W currently are the main observables for civilian navigation. S1C stands for the code C/A tracking at L1, S2W for the semi-codeless L2 P(Y) z-tracking, S2X for code M+L L2 tracking, and S5X for the code I+Q L5 tracking.

\section{Data and method}

GNSS data distributed in RINEX format contain S parameters (Pestana 2015), that represent the carrier-to-noise power density ratio (C/N0) or raw signal strength according to the RINEX 3.03 specification (ftp://igs.org/pub/data/format/rinex303.pdf ). The archive of the International GNSS service (IGS, Dow et al. 2019) at (ftp://igs.org/pub/station/general/igs08.atx) presents the schedule of the GPS satellite 
replacements. For the GPS satellite 10 (PRN10), the replacement of the IIA block with the IIF took place between August 3 and October 31, 2015.

In this work, we used data from the IGS station ZIM2 located in Switzerland $\left(7.47^{\circ} \mathrm{E}\right.$; $46.88^{\circ} \mathrm{N}$; Fig. 1). Figure 2 shows the $\mathrm{C} / \mathrm{N} 0$ changes for the GPS signal received at station ZIM2, at L1 (S1) and L2 (S2) on April 29, 2015 (blue dots) and December 27, 2015 (gray and black dots). These dates were specifically chosen outside the interval of the satellite replacement. It should be noted that on these days, there was no hardware update at the receiver. According to the official IGS information, during that time station ZIM2 was equipped with the Trimble NETR9 receiver and the TRM59800.00 antenna (http://www.igs.org/).
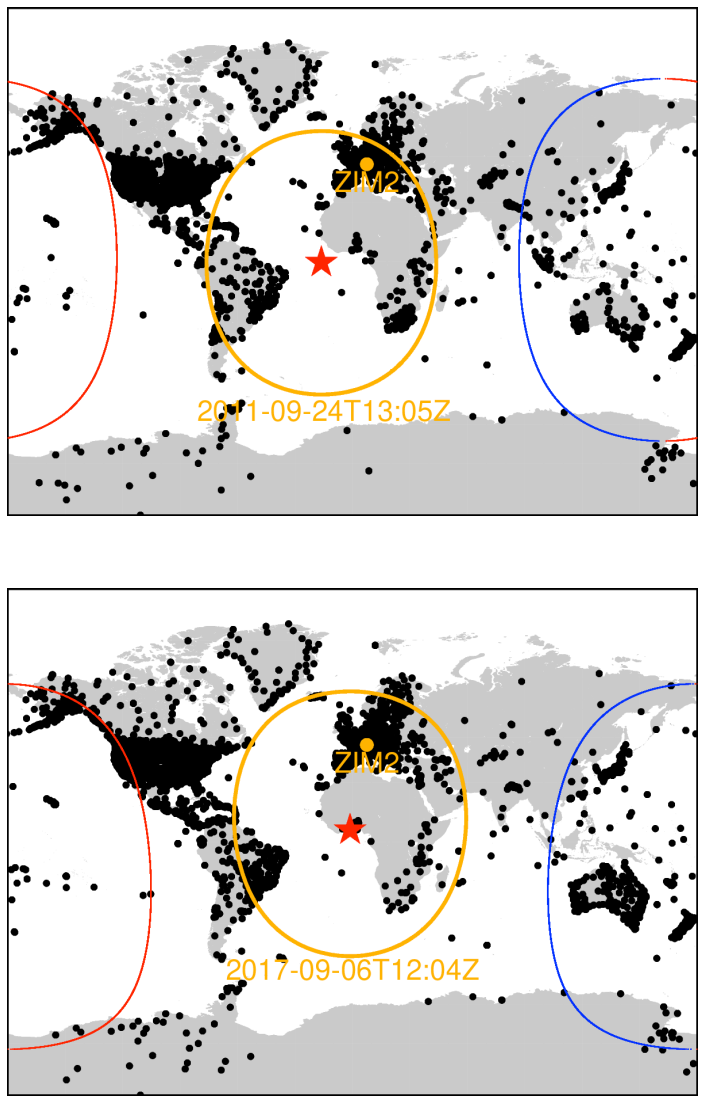

Fig. 1 Geometry of the experiment for the September 24, 2011 (top) and the September 06, 2017 (bottom) SRBs. The big orange dot shows the location of the IGS station ZIM2, the black dots show the worldwide GNSS networks, the red stars show the subsolar points during the maximum intensity of the solar flares, and the orange line confines the area with the solar zenith angle less than $60^{\circ}$ (solar elevation $>30^{\circ}$ ). Red and blue lines depict the position of the solar terminator at sunrise (red) and sunset (blue) at the moment of the SRBs maximum emission. 
The observables for station ZIM2 are taken from files in Rinex3 format. The files contain signal strength observables for the C/A code tracking and the semi-codeless $\mathrm{P}(\mathrm{Y}) \mathrm{z}$ tracking. We can? compare S2 observables at the same code (S2W) under z-tracking for both GPS IIA and GPS IIF. Also, we ean? estimate how the signal strength changes when we use the civilian $\mathrm{L} 2 \mathrm{C}(\mathrm{M}+\mathrm{L})$ tracking.

Figure 2 shows a change at $\mathrm{L} 1$ of about $1.5-2.5 \mathrm{~dB}-\mathrm{Hz}$ when receiving the signal from the modernized IIF satellite and a higher increase of about $3-4.5 \mathrm{~dB}-\mathrm{Hz}$ for $\mathrm{S} 2 \mathrm{~W}$ using $\mathrm{z}-$ tracking at L2. The signal strength increases by $8.5-20 \mathrm{~dB}-\mathrm{Hz}$ for the IIF-S2X (as compared to the IIA-S2W) using the $\mathrm{L} 2 \mathrm{C}(\mathrm{M}+\mathrm{L})$ tracking.
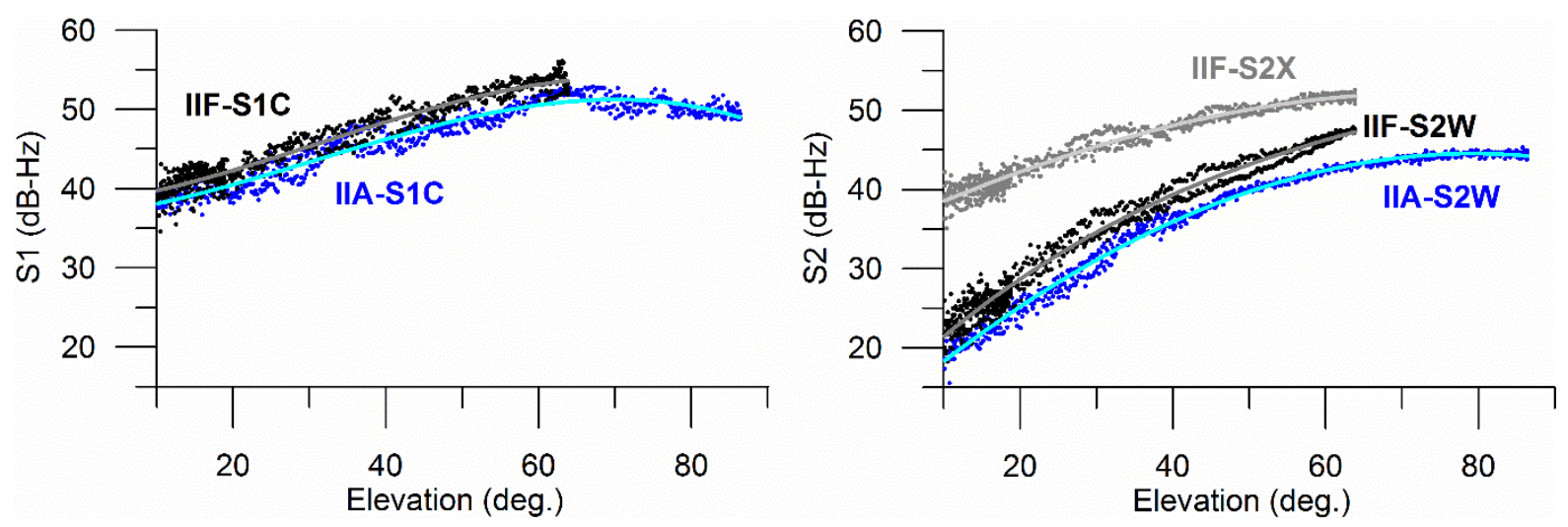

Fig. 2 Elevation angle dependence of GPS PRN10 received signal strength at L1 (left) and L2 (right) for April 29, 2015 (blue dots, GPS IIA) and for December 27, 2015 (gray and black dots, GPS IIF) at ZIM2 station. Black points are for the S1C (left) and S2W (right) from GPS IIF, blue points are for the S1C (left) and S2W (right) from GPS IIA, gray points are for the S2X from GPS IIF. Lines show the corresponding third order polynomial fits.

The best way to analyze the effect of the boost in the signal strength would be to compare the received signal power from one satellite during several radio bursts of similar intensity. However, this approach is difficult to implement because powerful radio bursts occur rarely. By analyzing the data over the recent 20 years, Huang et al. (2018) found that, within this period, there occurred only 2384 flares in the L-range, of which only 17 may be classified as severe $(10,000<$ radio emission flux $\leq 100,000 \mathrm{sfu})$ and 4 as extreme (radio emission flux $>100,000 \mathrm{sfu}$ ). Therefore, in this work, we implement the following approach: 

6, 2017 (Yasyukevich et al. 2018a; Berdermann et al. 2018) radio bursts that, according to Huang et al. (2018), are regarded as extreme and severe bursts, respectively. For each flare, we studied the dynamics for the received signal strength at the main (L1) and auxiliary (L2/L5) GPS frequencies at station ZIM2. The analysis was performed for differentgeneration satellite blocks. We used the S1C and S2W observables, which can be obtained from both "old" GPS block IIA/IIR-A and "new" GPS block IIR-M/IIF. Also, we used S2X observables to estimate code tracking performance at L2, and some data on S5X at L5. A Trimble NETR5 receiver was first installed at ZIM2, however, from April 27, 2015, the Trimble NETR9 version was in operation.

\section{Solar radio burst of September 24, 2011}

214 To analyze the SRB variations, we used data from the Sagamore Hill spectrograph that is a part of the RSTN international network (ftp://ftp.ngdc.noaa.gov/STP/space-weather/solardata/solar-features/solar-radio/rstn-1-second/sagamore-hill/). The September 24, 2011 radio emission at $1415 \mathrm{MHz}$ started rising at about 12:32 UT and peaked (114 $144 \mathrm{sfu}$ ) at 13:05 UT (Fig. 3, red line). Figure 3 shows that the signal strength decreased during the radio burst at the receiver at both L1 (S1) and at L2 (S2) frequencies. According to the RINEX 3.03 specification (ftp://igs.org/pub/data/format/rinex303.pdf) a threshold for good signal tracking is defined as $30-35 \mathrm{~dB}-\mathrm{Hz}$. For L1, the $\mathrm{C} / \mathrm{N} 0$ value threshold for the phase lock to occur, is about $28 \mathrm{~dB}-\mathrm{Hz}$ (Hofmann-Wellenhof et al. 2008). However, special signal post-processing procedures could lower this value to $20 \mathrm{~dB}-\mathrm{Hz}$ (Psiaki 2001). Therefore, in this work, we take $20 \mathrm{~dB}-\mathrm{Hz}$ as the threshold level.
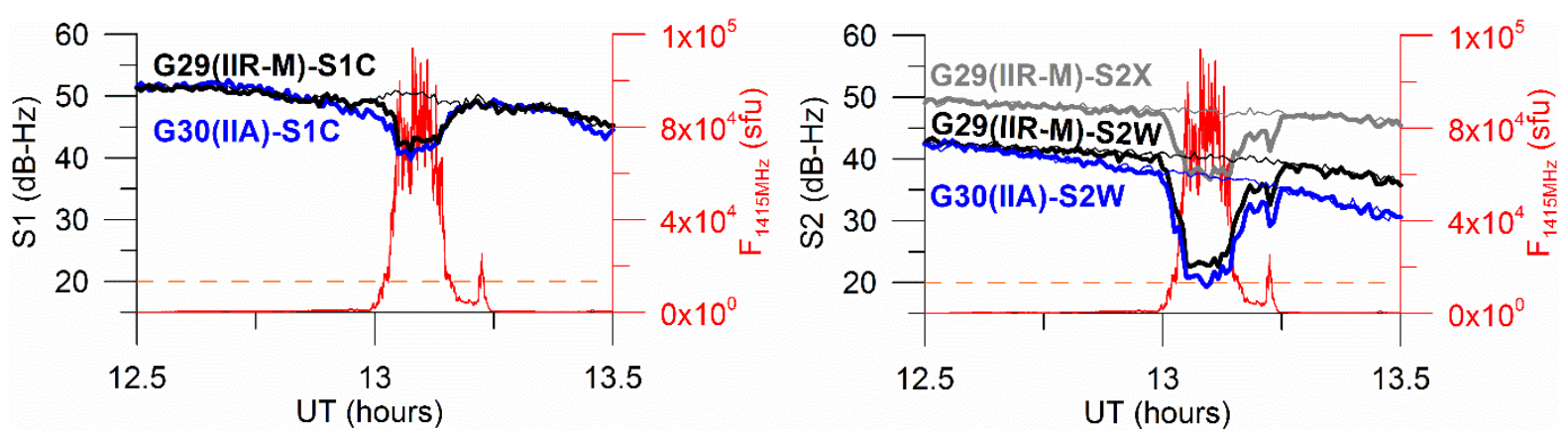

225 UT (hours) 
Fig. 3 Solar radio emission at $1415 \mathrm{MHz}$ as recorded by the Sagamore Hill radio

227

228

229

230

231

232

233 spectrograph (red curve) during the September 24, 2011 radio burst, and the signal strength (thick lines) at L1 (left panel) and L2 (right panel) at station ZIM2 for two satellites: G29 (IIR-M) and G30 (IIA). Black curves correspond to S1C and S2W, blue curves - to S1C and $\mathrm{S} 2 \mathrm{~W}$, and gray curves show S2X. Orange dashed line shows a $20 \mathrm{~dB}-\mathrm{Hz}$ threshold. Thin lines show the signal strengths for reference day (September 23). The data for the reference day are shifted by 4 minutes in order to obtain the same geometry of GPS sounding. Note that the same 4-minute time shift is applied for all reference day data throughout the article.

At L1, the received signal strengths S1C for both satellites have similar levels, whereas, at L2, the IIA S2W is lower than that at the IIR-M. During the flare peak, the IIA L2 received signal strength $\mathrm{S} 2 \mathrm{~W}$ drops below $20 \mathrm{~dB}-\mathrm{Hz}$, whereas, for the IIR-M, the minimum values of the received signal strength $\mathrm{S} 2 \mathrm{~W}$ is by $5 \mathrm{~dB}-\mathrm{Hz}$ higher.

Because the elevation angles for the satellites differ, it is better to compare the received signal strength to the elevation angle (as it was made in Fig. 2). Such comparison is presented in Figure 4 for L1 (S1, left) and for L2 (S2, right). One can see that, for L1, the dependence of the received signal strength S1C on the elevation angle is almost similar for the two blocks. During the flare, we observe a sudden drop of $\sim 7.5 \mathrm{~dB}-\mathrm{Hz}$ in the signal strength S1C. Such a drop occurs at different satellites for similar elevation angles. However, in general, the received signal strength remains sufficiently high. For L2, however, we observe a difference between the received signal strength for the two blocks: for IIA, the strength level is lower at all elevation angles. During the flare, we observe a decrease in the received signal strength $\mathrm{S} 2 \mathrm{~W}$ by more than about $17 \mathrm{~dB}-\mathrm{Hz}$ for both blocks. Due to the initially lower signal power, the IIA minimal value appears below the $20 \mathrm{~dB}-\mathrm{Hz}$ threshold, which potentially leads to a greater probability of the signal loss of lock during flares. Meanwhile, the decrease in the S2X for the PRN29 (the new block IIR-M) is only of $10 \mathrm{~dB}-$ $\mathrm{Hz}$.
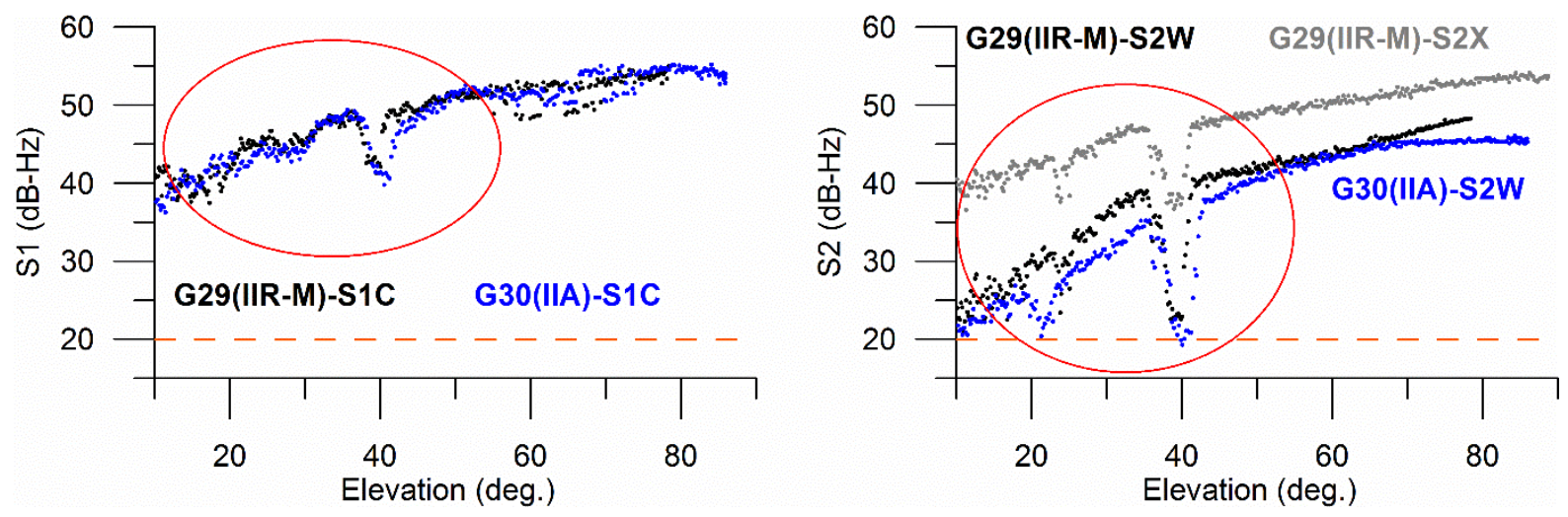
253 Fig. 4. GPS received signal strength at station ZIM2 at L1 (left panel) and L2 (right panel) 254 during the September 24, 2011 radio burst for two satellites: PRN29 (IIR-M, black and gray 255 dots), PRN30 (IIA, blue dots). Black dots correspond to the S1C (left panel) and to S2W 256 (right panel), blue dots are for S1C (left panel) and S2W (right panel), gray dots are for 257 S2X. Orange dashed line shows the $20 \mathrm{~dB}-\mathrm{Hz}$ threshold.

Thus, during the flare, we observe a sharp drop in the signal strength of about $7.5 \mathrm{~dB}$ $\mathrm{Hz}$ at $\mathrm{L} 1$ frequency and of $\sim 17 \mathrm{~dB}-\mathrm{Hz}$ at L2 frequency when using $\mathrm{P}(\mathrm{Y})$ code. However, due to the initially higher L2 signal power on satellites of new type, the signal strength during the flare exceeds the strength of the signal from the "old" satellite. This potentially results in more stable navigation. The flare-induced drop in the signal strength at L2 frequency when using the new civil code is smaller and is comparable with the drop at L1 frequency (about 10 $\mathrm{dB}-\mathrm{Hz})$.

\section{Solar radio burst of September 6, 2017}

268 The September 6, 2017 radio burst accompanied the most powerful X9.3 flare in the cycle 24 . 269 The maximum value of the radio emission flux reached $19604 \mathrm{sfu}$ at 12:04 UT as from the 270 San Vito radio spectrograph that is a part of the RSTN (ftp://ftp.ngdc.noaa.gov/STP/spaceweather/solar-data/solar-features/solar-radio/rstn-1-second/san-vito/).

Figure 5 shows the dynamics of the radio emission flux (red curve). The gray curve 273 shows variations in the received signal strength at ZIM2 from the GPS satellite PRN08 (IIF); the blue one does the same for the GPS PRN11 (IIR-A). One can see that this flare did not affect the measured signal strength $\mathrm{S} 1 \mathrm{C}$ at $\mathrm{L} 1$. At the same time, the flare caused $8 \mathrm{~dB}-\mathrm{Hz}$ decrease in the signal strength S2W at L2. This result corresponds to those obtained by Sato et al. (2019) who revealed a decrease in L1, L2, and L5 signal strength up to $10 \mathrm{~dB}-\mathrm{Hz}$ in the European region. A decrease in L2 signal strength using civilian L2C code (S2X) is slightly smaller - about $7 \mathrm{~dB}-\mathrm{Hz}$. 

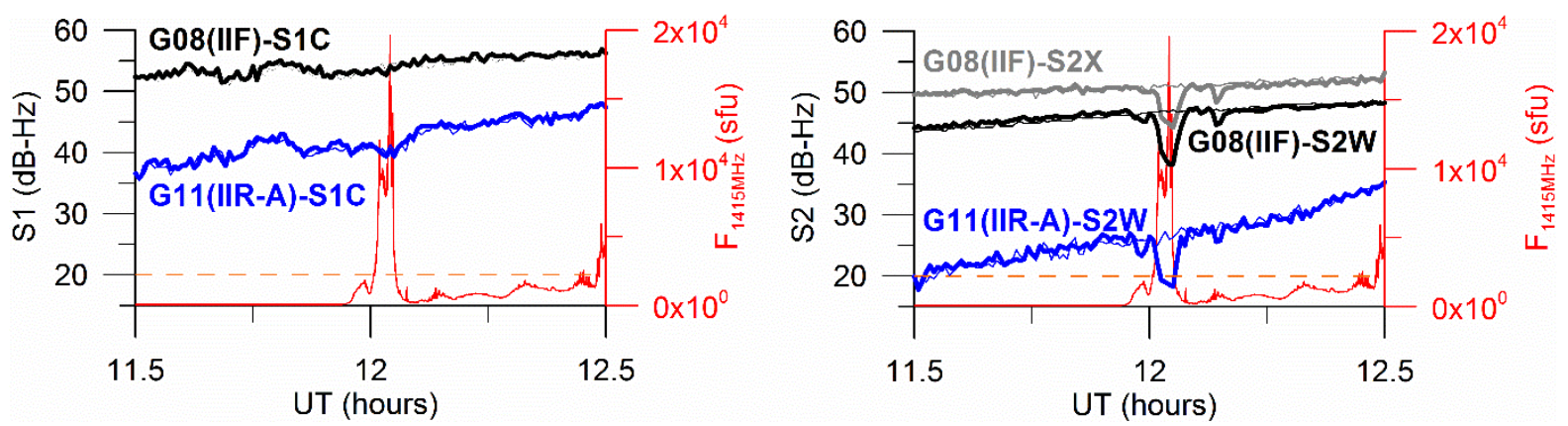

282 Fig. 5 Dynamics of the solar radio emission at $1415 \mathrm{MHz}$ as recorded by the San Vito spectrograph (red curve) during the September 6, 2017 radio burst and the GPS received signal strength (thick lines) at station ZIM2 for two satellites: G08 (IIF) and G11 (IIR-A).

The black curves are for S1C and S2W, blue curves are for S1C and S2W, and the gray curve 286 is for S2X. Thin lines are the signal strengths for reference day (September 5). Orange dashed line shows the $20 \mathrm{~dB}-\mathrm{Hz}$ threshold.

Figure 6 shows the dependence of the received signal strength on the elevation angle. Unfortunately, during the flare, there were no satellites of different types at similar elevations. However, one can see significant differences in the signal-to-noise ratio at L2. The solar flare effect is in a sharp decrease in the received signal strength at L2. The PRN11 (IIR-A) initially shows lower values for the L2W signal strength (Fig.6, right panel). During the flare, the signal strength dropped down to the $20 \mathrm{~dB}-\mathrm{Hz}$ threshold level. Note, that for the PRN08 (IIF), the radio burst effect was recorded at a higher elevation angle, therefore, a direct comparison of the signal strength from those satellites during the flare incorrect. Note, however, that the flare-driven total signal strength $\mathrm{S} 2 \mathrm{~W}$ decrease for both satellites is comparable by amplitude, i.e., $\sim 8 \mathrm{~dB}-\mathrm{Hz}$, and does not change with elevation. Therefore, if the radio burst occurred for the PRN08 at the same low elevation angle as for the PRN11, the minimal threshold for PRN08 signal strength would not be reached due to the initially higher power of the L2 P(Y) signal at PRN08. Signal strength for S2X at PRN 08 is higher, especially at low elevation angles and the flare-related effect for this signal is weaker.

Thus, increasing the power of the L2 signal emitted from a satellite can improve the stability of the system against the solar flare threat. However, the use of the new open civil 305 code contributes even greater to the enhancement in the system stability. 

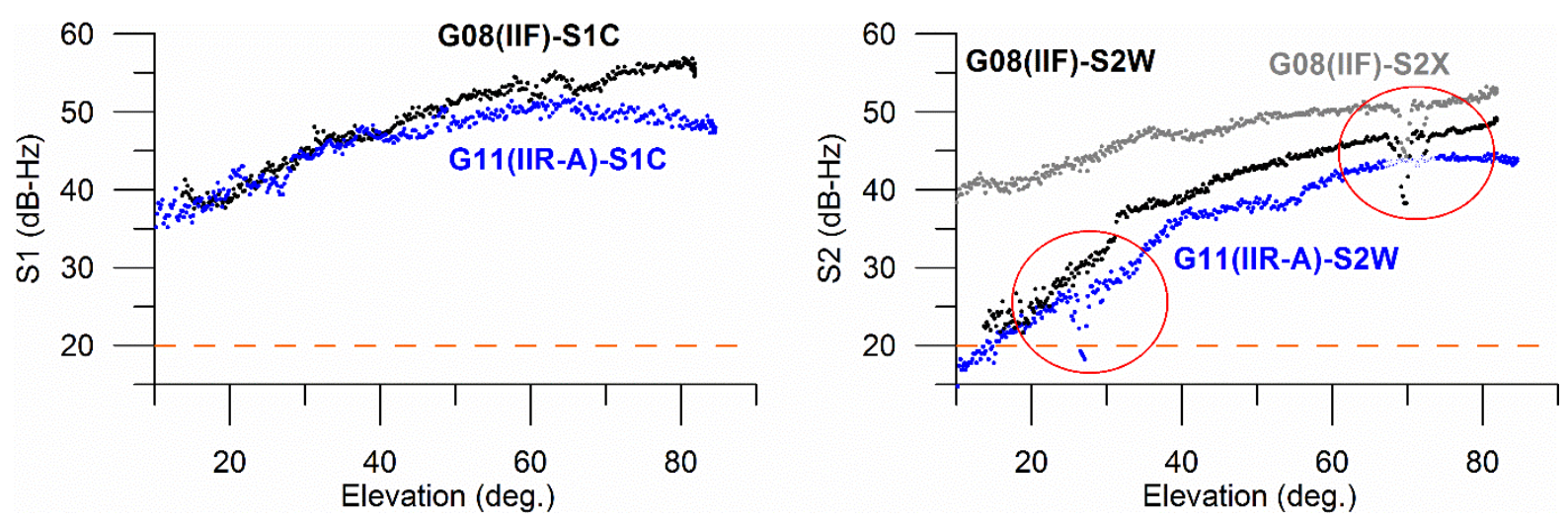

308

Fig. 6. GPS received signal strength at ZIM2 at L1 (left) and L2 (right) during the September 6, 2017 radio burst for two satellites: PRN08 (IIR-M, black and gray dots), PRN30 (IIR-A, blue dots). Black dots are for S1C (left) and S2W (right), blue dots are for S1C (left panel) and S2W (right), gray dots are for S2X. Orange dashed lines show the $20 \mathrm{~dB}-\mathrm{Hz}$ threshold.

The GPS IIF is equipped with a transmitter emitting at L5 frequency. This enables us to analyze the flare effect on different frequencies for the modernized blocks. Figure 7 shows the dynamics for the GPS received signal strength at L1 (S1, black), L2 (S2, gray), L5 (S5, navy blue). The data are for station ZIM2 except for the L5 data in 2011, when ZIM2 did not record L5 observables. Thus, we find another station WTZR $\left(49.14^{\circ} \mathrm{N} ; 12.87^{\circ} \mathrm{E}\right)$ and put this data on the left panel (see S5-WTZR). In 2011, a Leica GRX1200+GNSS receiver with the LEIAR25.R3 LEIT antenna was installed at station WTZR. However, the WTZR data are only provided in RINEX2 format, therefore we do not have detailed information on observables S5Q, S5I or S5X. In 2011, only two satellites were equipped with the IIF blocks: PRN01 and PRN25. PRN01 was not visible in the region during the flare, while signals from PRN25 were captured at a low elevation of about $15^{\circ}$. Therefore, we used observations S1C, S2W, S2X from PRN29 (IIR-M), and adjusted S5 from PRN25 (IIF). To adjust S5 measurements, we increased all S observables from PRN25 by $5 \mathrm{~dB}-\mathrm{Hz}$ to match S1C level from PRN29 and PRN25.

Figure 7 shows the strength level of L5 and L1 signals. While we do not observe any decrease in the signal strength in the September 6, 2017 S1C data, the L5 signal strength drop of $\sim 7.5 \mathrm{~dB}-\mathrm{Hz}$ is comparable, although less pronounced, with the L2 power loss. Our finding agrees the results of Sato et al. (2019), who reported the impact of the September 6, 2017 SRB at GPS L2 and L5 frequencies, but not at L1 frequency. Nevertheless, the use of code tracking (L5 and L2C) decreases the signal strength as compared to semi-codeless tracking (P(Y)). For instance, during the September 24, 2011 SRB, the L5 signal strength S5 dropped 
by $9 \mathrm{~dB}-\mathrm{Hz}$. Among the considered examples, the $\mathrm{P}(\mathrm{Y})$ signal (S2W observable) shows the

September 24, 2011

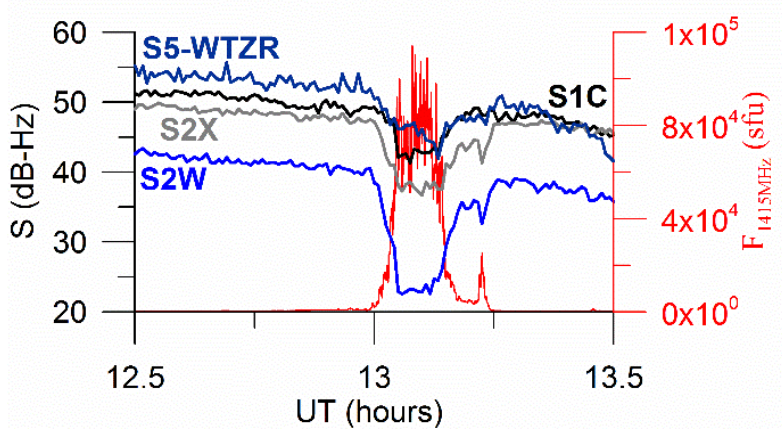

September 06, 2017

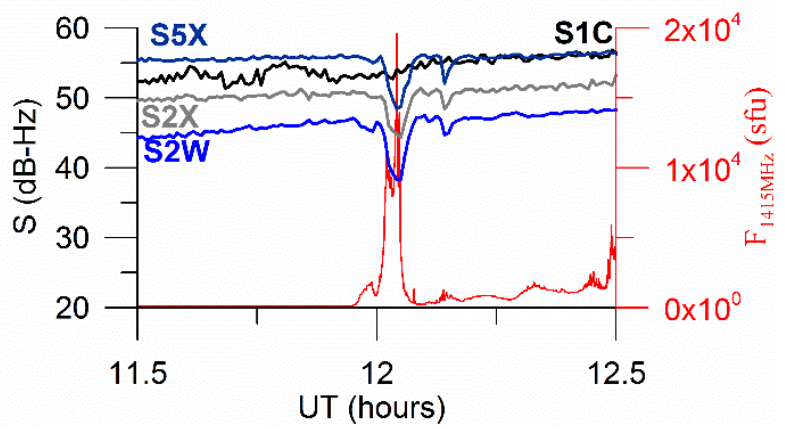

338

Fig. 7 Dynamics of the strength of GPS received signal S1C (black lines), S2W (blue lines), S2X (gray lines), S5X (navy blue) at station ZIM2. The solar radio emission data for the September 24, 2011 (left panel) and for the September 6, 2017 (right panel) solar flares are shown in red. On the left panel, we show S5 observable at station WTZR (S5-WTZR, navy blue).

\section{LoL dynamics during the flares}

A decrease in the received signal strength during solar radio bursts often leads to a greater probability of signal losses of lock (e.g., Afraimovich et al. 2006). Here, we analyze how the boost of the emitted signal power at satellites of the new generation affected the general stability of the system operation during solar radio bursts. For this purpose, we estimate the mean density of the GPS signal phase LoL for L1 and L2 during the considered flares. Our results are based on data of the global GNSS network that included about 5000 (4659 in 2011 and 4939 in 2017) receivers. The global network includes different global and regional networks such as IGS (Dow et al. 2009), UNAVCO, SONEL, CHAIN (Jayachandran et al. 2009), EUREF (Bruyninx et al. 2012), and SibNet (Yasyukevich et al. 2018b). Fig. 1 shows the distribution of GPS stations that we used in this study. In 2011, data for almost all receivers were provided only in Rinex 2 format without information on code tracking type. In 2017, most receivers kept data only in Rinex2 format. Most likely, Rinex2 files contained S1C as S1 and S2W as S2, because if it contained S2X/S2L/S2M as S2, then there was a lot of missing data for "old" GPS satellites which do not emit modernized signals. Wanniger (2018) found that $2.7 \%$ of GPS receivers mix L2P(Y)/L2C observations in the 
Rinex files. We assume that $2.7 \%$ is not high enough and should not significantly change the global LoL pattern. Thus, we believe that in our study the observed differences in the LoL density dynamics for "old" and "new" satellites are associated with the improved emitted signal power rather than with the use of different codes.

We calculated the LoL relative density $P_{T}$. For this purpose, at a station, the number of LoL $\mathrm{N}(\mathrm{t})$ within the selected time interval $t$ was normalized for the total number of observations $\mathrm{S}(\mathrm{t})$. Then, we averaged over all the GPS satellites recorded within this interval $(M)$. Further, we calculated the LoL relative density (percent):

$$
P_{T}=\frac{1}{M} \sum_{j=1}^{M} \frac{N(t)_{j}}{S(t)_{j}}
$$

To estimate the LoL density, we used data from stations located close to the subsolar region at the flare instant. For this purpose, in formula (1), we consider only ionospheric pierce points (a point of line-of-sight (LOS) intersection with the ionosphere at $300 \mathrm{~km}$ height) with the solar zenith angle below $60^{\circ}$ (solar elevation $>30^{\circ}$; this area is marked by orange lines in Fig.1). Finally, we selected only LOS with elevation angles higher than $10^{\circ}$. As a result, we calculated the LoL density separately for the modernized and "old" satellites. At each time instant, the statistics included more than 3000 records for both flares and for both types of satellites.

Fig. 8. shows the estimated LoL densities. At all satellites, the LoL density at L2 significantly exceeded that at L1 during the flares. In both cases, sharp peaks in the LoL density dynamics accompanied the radio bursts (vertical dashed lines). We do not recorded such peaks on previous quiet days.

During the 2011 extreme radio burst, a sharp increase in the LoL density at L2 reached $30 \%$ of the total number of measurements at the "old" satellites (Fig. 8 bottom left). At the newer satellites, the LoL density maximum value at L2 reached 10\% (Fig. 8 top left), i.e., $20 \%$ lower than for the "old" satellites. At L1, the maximum LoL density during the flare grew up to $0.6 \%$ for modernized satellites and exceeded $1 \%$ for the "old" ones. Overall, one may state an essential degradation of the system operation quality during this event. However, the increased power at the modernized satellites reduced susceptibility to SRB even under semi-codeless treatment. 
a

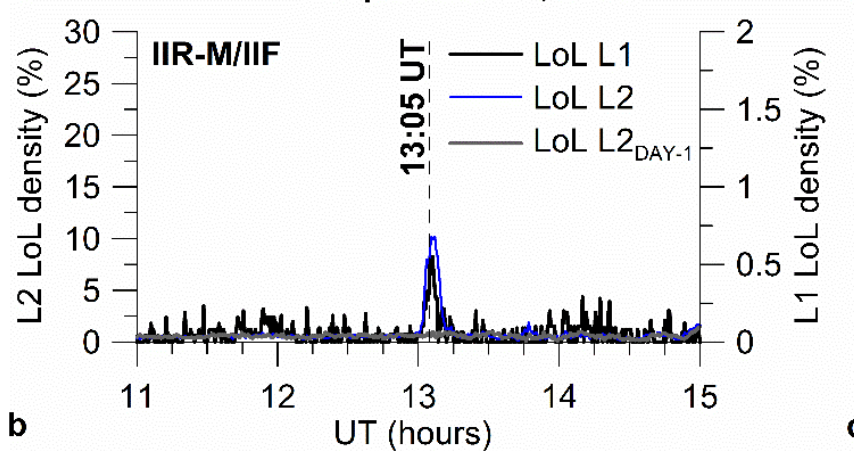

c

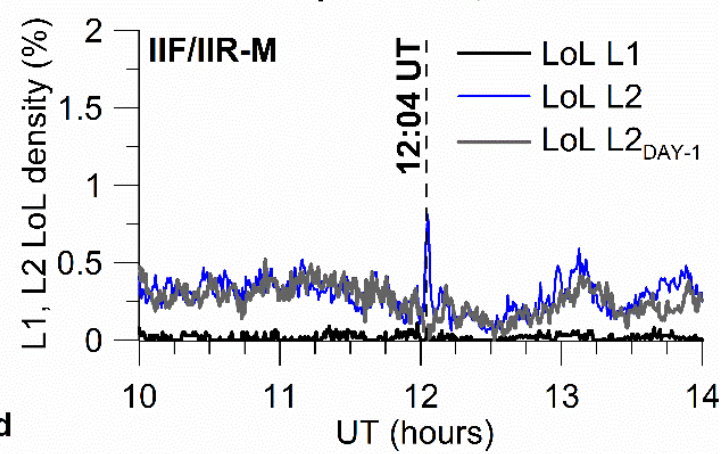

390

391

392

393

394

395

396

397

398

399

400

401

402

403

404

405

406

407

408

409

410

411
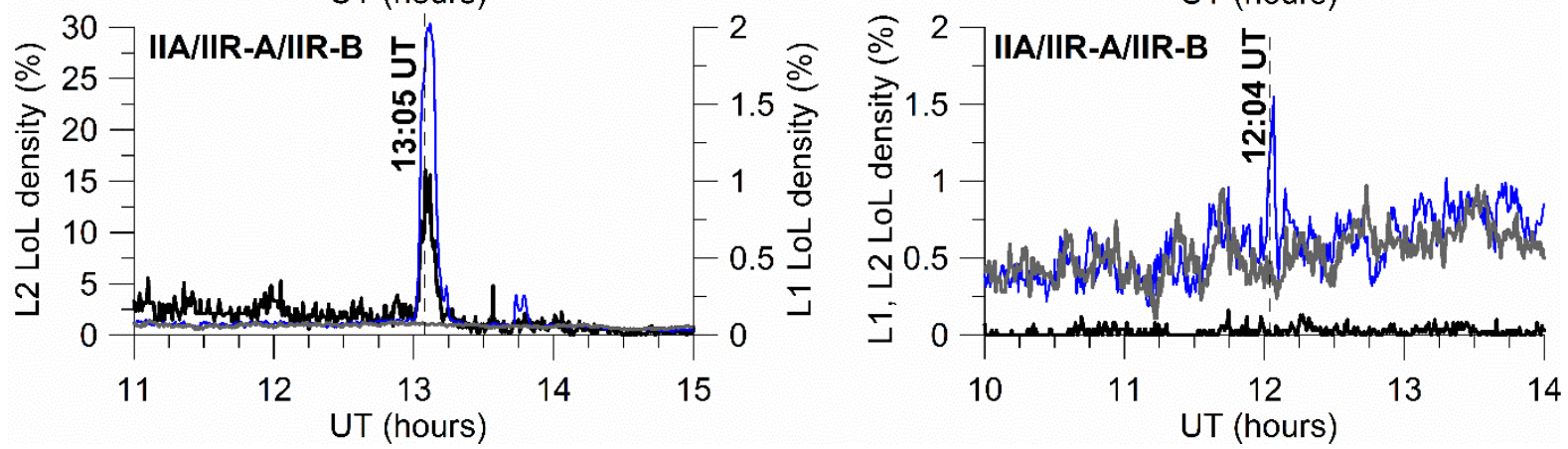

Fig. 8 Density of losses of lock at L1 (black curve) and L2 (blue curve) in the subsolar region during the September 24, 2011 (left) and the September 6, 2017 (right) solar radio bursts for the modernized (top) and "old" (bottom) satellites. Right scales on the left panels are for L1 LoL density in 2011. Bold gray lines show the dynamics of L2 LoL density on previous days. Vertical dashed lines depict the SRBs maxima. Note that for the September 24, 2011 SRB (left panels), we selected the Y-scales for L1 and L2 so that the LoL density levels on the previous day coincided (gray line, top left).

Fig. 8 shows a flare related LoL density increase in 2017, but to a much lesser extent as compared to those in 2011. The maximal values of the LoL density at L2 during the flare reached about $1.6 \%$ at the "old" satellites (bottom right), and about $0.8 \%$ for modernized satellites (top right). There was no distinguished effect on L1 LoL density dynamics for this flare. Lesser LoL density during the 2017 SRB is caused, first of all, by a lower intensity of the radio burst. Another factor is the different duration of the disturbance impulse during the flares. So, in 2017 the radio flux exceeded a relative threshold value of 5000 sfu for about 2 minutes. In 2011 the threshold was surpassed for more than 10 minutes with short second peak of about 1 minutes. It indicates a strong difference between the total disturbing energy amount injected during the flares.

Losses of lock during the September 6, 2017 flare over the European region were studied in detail by Berdermann et al. (2018). Their results indicated that "the space weather event does not coincide with the loss of lock intervals". A smaller number of stations (as compared to the current research) and possibly their better performance could result in the 
inconsistency with our results shown in Fig. 8. It is interesting to note that Yasyukevich et al. (2018a) found no LoL during the September 2017 flare either. Possible reason for this difference in our results with previous ones could be a different set of stations. The main advance of the current approach is introducing a limit on the solar zenith angle. As we can see from Fig. 1 there are a lot of GPS stations in Europe and America, which are on the sunlit part of the Earth. However, most of these stations correspond to low solar elevation $\left(<60^{\circ}\right)$. At stations with low solar elevation, SRB did not impact the losses-of-lock. Therefore, in previous studies, adding stations with low elevation could "hide" the effect. Our new approach allowed us to reveal the effect.

Both the satellite elevation and solar zenith angle should influence the loss of lock density: lower signal strength at low elevation increases LoL probability, higher emission flux at low zenith angles does the same. Top panel in Fig. 9 demonstrates the total LoL density at L2 depending on the elevation and solar zenith angle during the 10-min period around the peak of the 2011 SRB. The figure confirms that during the flare most of LoLs appear at low zenith angles, generally less than $40^{\circ}$. Interesting to note, that for this powerful flare there were losses of lock at zenith angles around $30^{\circ}$ even at high elevations.

Middle and bottom panels in Fig. 9 show the LoL density dynamic at L1 (black curves) and L2 (blue curves) for the modernized (solid lines) and "old" (dashed lines) satellites. LoLs are calculated for the 10-min periods around the peaks of the September 24, 2011 (middle) and the September 6, 2017 (bottom) solar radio bursts, correspondingly. We do not show here LoL dynamics at L1 during the 2017 SRB, because a small number of the LoLs recorded. This did not allow us to get a reliable dependence. During the both flares LoL density at L2 (for modernized and "old" satellites) depends on solar zenith angle. At L1 in 2011 this dependency is not clear enough. We could use the dependencies to predict losses of phase lock probability under SRB. The supplementary materials to the article contains the temporal evolution of the LoLs depending on the solar zenith angle at both modernized and “old" satellites during the flares. The figures additionally confirm mentioned above.

In both cases, the L2 LoL density decreased more than twice at modernized satellites. Hence, one may conclude that the increasing emitted signal power at modernized satellites improves the system stability with respect to both severe and extreme solar radio bursts. 
September 24, 2011
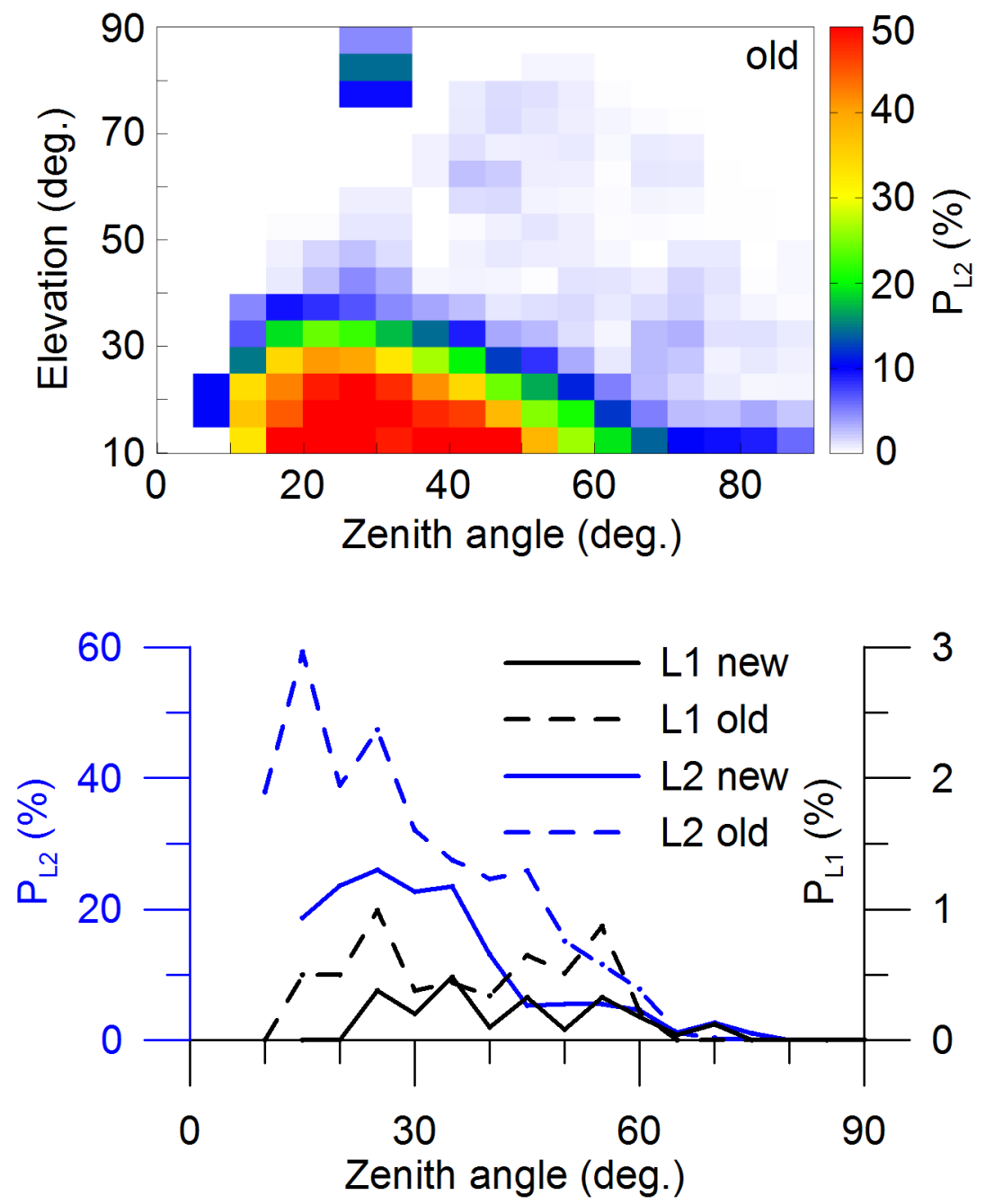

September 6, 2017

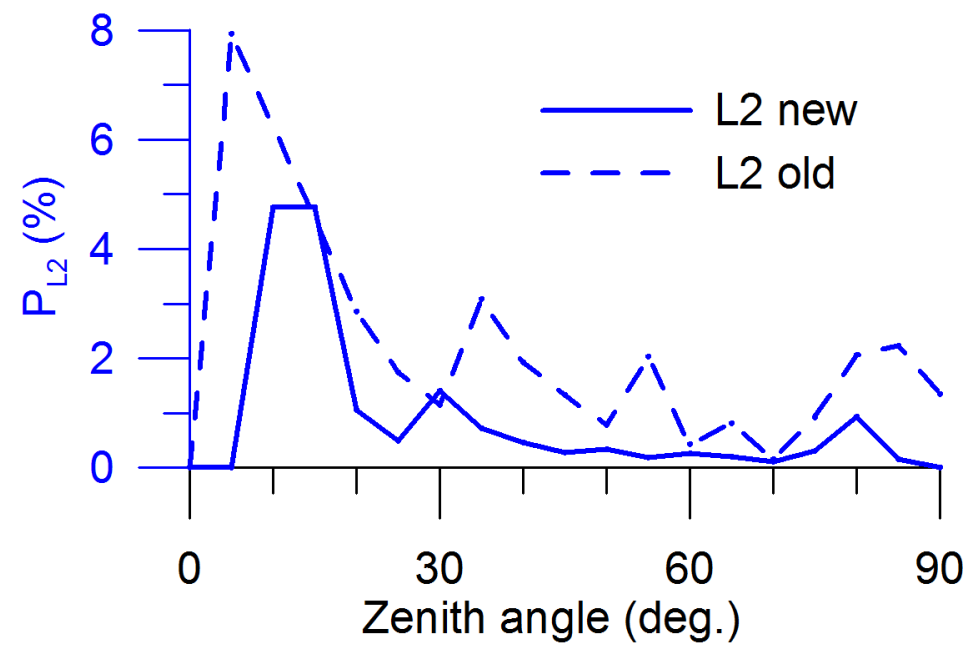

Fig. 9 Loss of lock density vs. the satellite elevation and the solar zenith angle. Top 444 panel shows 2D-distribution of LoL density at L2 vs. the elevation and the solar zenith angle 445 for the 2011 solar radio burst. Middle and bottom panels shows LoL density dynamics vs. the 446 solar zenith angle for the 2011 SRB and the 2017 SRB, correspondingly: black marks L1 447 data, blue marks L2 data, solid lines corresponds to the modernized satellites, and dashed 
lines correspond to "old" satellites. Calculations are for the 10-min periods around the SRB peaks.

\section{Summary and conclusion}

451

In this work, we analyzed how the increase in the emitted signal power at modernized GPS satellites affected the system operation stability during the extreme (September 24, 2011) and severe (September 6, 2017) solar radio bursts. We showed that, after the IIA/IIR-A blocks were replaced with IIR-M/IIF modernized ones, the signal strength S2W at L2 frequency increased by about 3-4.5 dB-Hz at station ZIM2. Even a stronger increase in signal power was observed for the modernized L2C code (S2W observables): the increase reached $20 \mathrm{~dB}-$ $\mathrm{Hz}$ compared with the IIA S2W. During the considered solar radio bursts, the navigation signal strength decreased at L2 and L5 for semi-codeless Z-tracking (S2W) and code tracking (S2X, S5X). Herewith, the L2 signal under semi-codeless P(Y) z-tracking features the least total power ( $\mathrm{S} 2 \mathrm{~W}$ ) and, thereupon, the worst stability during the radio bursts. Also, it is $\mathrm{S} 2 \mathrm{~W}$ that corresponds to the maximum drop of signal strength during SRB. During the 2011/2017 SRBs the signal strength drops were: 7.5/0 dB-Hz for S1C, 10/7 dB-Hz for S2X, 9/7.5 for S5/S5X, 17/8 dB-Hz for S2W for the corresponding SRBs peaks. Thus, more intensive SRB corresponds to a higher drop in signal strength. We should note that using civilian codes enables us to decrease the signal strength drop under solar radio bursts.

Drop in the signal strength $\mathrm{S} 2 \mathrm{~W}$ for the modernized blocks was comparable in amplitude to those of "old" blocks. However, IIR-M/IIF recorded about $5 \mathrm{~dB}-\mathrm{Hz}$ higher signal strength. This difference does not depend on the receiver and antenna type. This potentially results in more stable navigation.

By analyzing the GPS L1/L2 signal loss of locks during both SRBs we showed that the LoL density at L2 at modernized satellites was lower than that at the "old" satellites. During the extreme SRB, the LoL density at "old" satellites reached 30, which is 3 times higher than at modernized satellites. The corresponding values for the severe SRB were 1.6\% and 2 times. Therefore, enhanced emitted signals improve GPS signal tracking. During the September 24, 2017 extreme SRB we also detected an increase in LoL density at L1 frequency for both types of satellites. Meanwhile, there was no obvious effect on L1 LoL density during the September 6, 2017 severe SRB.

We must emphasize that we did not compare the effects of the 2011 and 2017 flares in our study. For each case, we separately analyze the stability of signals from "old" and "new" satellites received at the same station (respectively, on the same equipment and software). 
Also, we do not aim to determine which mechanism - SITEC or SRB- is prevalent in producing the LoLs during the flares. Therefore, without entering into an argument about the main impact, our results prove that the increase in the emitted signal power at modernized satellites improves the system stability with respect to the threats from both severe and extreme flares accompanied by solar radio bursts. Modernized signals also can improve the stability.

\section{Acknowledgments}

We thank Dr. I. Zhivetiev for the "tec-suite" software (http://www.gnss-lab.org/) used for RINEX files processing. We acknowledge the organizations providing GNSS data. This work was performed with the support of the Russian Federation President Grant No. MK3265.2019.5 and the budgetary funding of Basic Research program II.16.

\section{Data Availability}

The solar radio spectrographs data are publicly available from the National Geophysical Data Center (NGDC, http://www.ngdc.noaa.gov/ngdc.html); station ZIM2 data are available from the EUREF Permanent Network Services (ftp://ftp.epncb.oma.be/). We used GNSS data obtained and publicly stored by the Scripps Orbit and Permanent Array Center, UCSD (ftp://garner.ucsd.edu/; http://lox.ucsd.edu/pub/), Bundesamt für Kartographie und Geodäsie Data Center (ftp://igs.bkg.bund.de/), Wuhan University (ftp://igs.gnsswhu.cn/), Crustal Dynamics Data Information System (ftp://cddis.gsfc.nasa.gov/), Korea Astronomy and Space

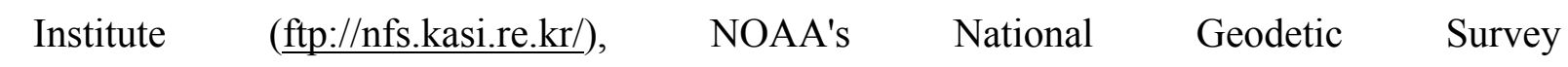
(ftp://www.ngs.noaa.gov/cors), UNAVCO (ftp://data-out.unavco.org/), Système d'Observation du Niveau des Eaux Littorales (SONEL, ftp://ftp.sonel.org/), the EUREF Permanent Network Services (ftp://ftp.epncb.oma.be/), Geoscience Australia (ftp://ftp.ga.gov.au/), the New Zealand GeoNet project (ftp://ftp.geonet.org.nz/), the State GPS network of the Republic of Bulgaria (ftp://195.96.249.3/), Institut Geographique National (ftp://igs.ensg.ign.fr/), Instituto Geográfico Nacional (ftp.geodesia.ign.es), Instituto Tecnológico Agrario de Castilla y León (ftp.itacyl.es), Geodetic Data Archiving Facility (ftp://geodaf.mt.asi.it/), and Instituto Brasileiro de Geografia e Estatística (ftp://geoftp.ibge.gov.br/). Data of Canadian High Arctic Ionospheric Network 
512 (http://chain.physics.unb.ca/) and data of SibNet network as a part of Center for Common 513 Use "Angara” (http://ckp-angara.iszf.irk.ru/) are available upon request.

References performance in geomagnetically disturbed conditions. GPS Solut. 7(2):109-119. https://doi.org/10.1007/s10291-003-0053-7

Afraimovich EL, et al. (2013) A review of GPS/GLONASS studies of the ionospheric response to natural and anthropogenic processes and phenomena. J. Space Weather Space

Afraimovich EL, Demyanov VV, Smolkov GY (2009) The total failures of GPS functioning caused by the powerful solar radio burst on December 13, 2006. Earth Planets and Space, 61:637-641. https://doi.org/10.1186/BF03352940

Ashjaee J, Lorenz R (1992) Precision GPS surveying after Y-code. In: Proc. ION GPS 1992, Institute of Navigation, Albuquerque, NM, USA, September 16-18, 657-659. super-storms, and their impacts on GPS-based navigation systems. Space Weather, 12(7):508-525. https://doi.org/10.1002/2014SW001072

Berdermann J, Kriegel M, Banys D, Heymann F, Hoque MN, Wilken V, Borries C, Heßelbarth A, Jakowski N (2018) Ionospheric response to the X9.3 Flare on 6 September 2017 and its implication for navigation services over Europe. Space Weather, 16:1604-1615. https://doi.org/10.1029/2018SW001933 
Observed solar radio burst effects on GPS/ Wide Area Augmentation System carrier-to-noise ratio. Space Weather, 4(10):S10006. https://doi.org/10.1029/2006SW000254

Chen Z, Gao Y, Liu Z (2005) Evaluation of solar radio bursts' effect on GPS receiver signal tracking within International GPS Service network. Radio Science, 40:RS3012. https://doi.org/10.1029/2004RS003066

Coster A, Komjathy A (2008) Space weather and the Global Positioning System. Space Weather, 6:S06D04. https://doi.org/10.1029/2008SW000400

Demyanov VV, Yasyukevich YuV, Jin S (2013) Effects of solar radio emission and ionospheric irregularities on GPS/GLONASS performance. In: Geodetic Sciences https://doi.org/10.5772/3439

Dow JM, Neilan RE, Rizos C (2009) The International GNSS Service in a changing landscape of Global Navigation Satellite Systems. J. Geodesy, 83:191-198. https://doi.org/10.1007/s0019000803003

Fortes LPS, Lin T, Lachapelle G (2015) Effects of the 2012-2013 solar maximum on GNSS signals in Brazil. GPS Solut, 19:309-319. https://doi.org/10.1007/s10291-014-0389-1

Hofmann-Wellenhof B, Lichtenegger H, Wasle E (2008) GNSS - Global Navigation 558 Satellite Systems: GPS, GLONASS, Galileo \& more. Springer, Vienna. https://doi.org/10.1007/978-3-211-73017-1 radio bursts. GPS Solut., 22:114. https://doi.org/10.1007/s10291-018-0780-4

IS-GPS-200J. Global positioning systems directorate. Systems engineering \& 563 integration interface specification IS-GPS-200, 2018, https://gps.gov/technical/icwg/IS-GPS564 200J.pdf.

Jakowski N, Stankov SM, Klaehn D (2005) Operational space-weather service for 566 GNSS precise positioning. Annales Geophysicae, 23:3071-3079. 567 https://doi.org/10.5194/angeo-23-3071-2005 
Jayachandran PT, Langley RB, MacDougall JW, et al (2009) The Canadian high

arctic ionospheric network (CHAIN). Radio Science, 44:RS0A03.

570 https://doi.org/10.1029/2008RS004046

571

572

573

574

575

576

577

578

579

Jin Y, Oksavik K (2018) GPS scintillations and losses of signal lock at high latitudes during the 2015 St. Patrick's Day storm. J. Geophys. Res. Space Phys., 123:7943-7957. https://doi.org/10.1029/2018JA025933

Klobuchar JA, Kunches JM, Van Dierendonck AJ (1999) Eye on the ionosphere: Potential solar radio burst effects on GPS signal to noise. GPS Solut., 3(2):69-71. https://doi.org/10.1007/PL00012794

Matsoukas D, Papagiannis M, Aarons J, Klobuchar J (1972) Correlation of solar radio bursts and sudden increases of the total electron content (SITEC) of the ionosphere. J. of Atmos. and Terr. Phys., 34(7):1275-1283. https://doi.org/10.1016/0021-9169(72)90137-7

Muhammad B, Alberti V, Marassi A, Cianca E, Messerotti M (2015) Performance assessment of GPS receivers during the September 24, 2011 solar radio burst event. J. Space Weather Space Clim., 5:A32. https://doi.org/10.1051/swsc/2015034

Pestana A (2015) Technical Report: Reading RINEX 2.11 Observation Data Files. https://doi.org/10.13140/RG.2.1.4888.4087

Psiaki ML (2001) Block acquisition of weak GPS signals in a software receiver. In: Proc. ION GPS 2001, Salt Lake City, UT, September 11-14, pp 2838-2850.

Rodríguez-Bilbao I, Radicella SM, Rodríguez-Caderot G, Herraiz M (2015) Precise point positioning performance in the presence of the 28 October 2003 sudden increase in total electron content. Space Weather, 13:698-708. https://doi.org/10.1002/2015SW001201

Sato H, Jakowski N, Berdermann J, Jiricka K, Heßelbarth A, Banyś D, Wilken V (2019) Solar radio burst events on 6 September 2017 and its impact on GNSS signal frequencies. SpaceWeather, 17:816-826. https://doi.org/10.1029/2019SW002198

Sreeja V, Aquino M, Jong K (2013) Impact of the 24 September 2011 solar radio burst on the performance of GNSS receivers. Space Weather, 11:306-312. https://doi.org/10.1002/swe.20057 
radio burst on precise point positioning service. Space Weather, 12(3):143-147. https://doi.org/10.1002/2013SW001011

599

600

601

602

603

604

605

606

607

608

609

610

611

612

613

614

615

616

617

618

619

620

621

622

623

Tsurutani BT, Verkhoglyadova OP, Mannucci AJ, Lakhina GS, Li G, Zank GP (2009) A brief review of "solar flare effects" on the ionosphere. Radio Science, 44:RS0A17. https://doi.org/10.1029/2008RS004029

Wan W, Liu L, Yuan H, Ning B, Zhang S (2005) The GPS measured SITEC caused by the very intense solar flare on July 14, 2000. Adv. Space Res., 36:2465-2469. https://doi.org/10.1016/j.asr.2004.01.027

Wanninger L (2018) Detection of RINEX-2 files with wixed GPS L2P(Y)/L2C carrier phase observations. Sensors, 18(12):4507. https://doi.org/10.3390/s18124507

Yasyukevich Y, Astafyeva E, Padokhin A, Ivanova V, Syrovatskii S, Podlesnyi A (2018a) The 6 September 2017 X-class solar flares and their impacts on the ionosphere, GNSS, and HF radio wave propagation. Space Weather, 16:1013-1027. https://doi.org/10.1029/2018SW001932

Yasyukevich YuV, Vesnin AM, Perevalova NP (2018b) SibNet - Siberian Global Navigation Satellite System Network: Current state. Solar-Terr. Physics, 4(4):63-72. https://doi.org/10.12737/stp-44201809

Zakharov VI, Yasyukevich YuV, Titova MA (2016) Effect of magnetic storms and substorms on GPS slips at high latitudes. Cosmic Research, 54(1):20-30. https://doi.org/10.1134/S0010952516010147

Zhang DH, Xiao Z (2005) Study of ionospheric response to the 4B flare on 28 October 2003 using International GPS Service network data. J. Geophys. Res., 110:A03307. https://doi.org/10.1029/2004JA010738

Zhang SR, Coster AJ, Erickson PJ, Goncharenko LP, Rideout W, Vierinen, J (2019) Traveling ionospheric disturbances and ionospheric perturbations associated with solar flares in September 2017. J. Geophys. Res.: Space Phys., 124:5894-5917. https://doi.org/10.1029/2019JA026585 
626

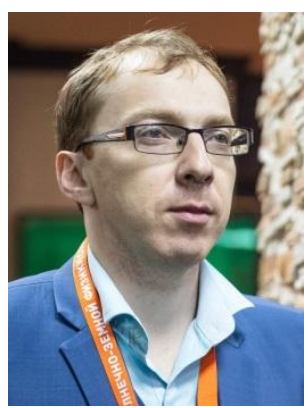

627

Yury V. Yasyukevich received his Ph.D. degree in Radio Physics from ISTP SB RAS in 2009. He works at the ISTP SB RAS as a leading researcher and the Irkutsk State University as an Associate Professor. His scientific interests include monitoring and modeling based on 630 GNSS data, space weather impact on GPS/GLONASS, estimating the absolute total electron content, and ionospheric anomalies. $\mathrm{He}$ is the PI of the SIMuRG project 632 (http://simurg.iszf.irk.ru).

633

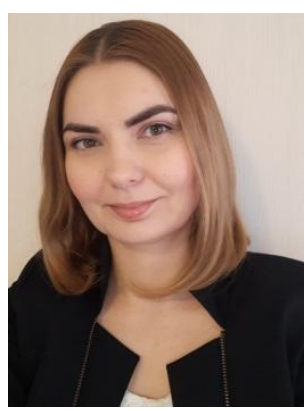

634

635

636

639 Elvira I. Astafyeva received her Ph.D. at the Institute of Solar-Terrestrial Physics (Siberian 640 Branch, Russian Academy of Sciences) in 2005. She has been a tenured CNRS researcher at 641

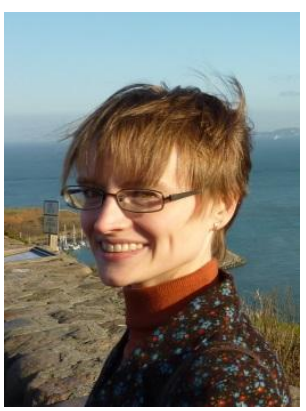

Anna S. Yasyukevich received her Ph.D. degree in Physics of the Atmosphere and Hydrosphere at the Institute of Solar-Terrestrial Physics SB RAS in 2015. She works at the ISTP SB RAS as a scientific researcher. Her scientific interests are space weather impact on GNSS, ionospheric morphology and neutral atmosphere-ionosphere coupling. the Institute of Physics of the Earth of Paris (IPGP) since 2012. Her main interests include 
642 ionospheric response to natural hazards, geomagnetic, ionospheric and thermospheric storms, 643 and space weather. 